ANALYTICAL LETTERS, 20(10), 1595-1610 (1987)

\title{
DETERMINATION OF THE CONCENTRATION OF GASES BY MEASUREMENT OF PRESSURE
}

\begin{abstract}
KEY WORDS: Determination of Concentration, Gases, Equation of State, Pressure Measurement
\end{abstract}

\author{
H. Langhals \\ Institut für Organische Chemie \\ der Universität München \\ Karlstraße 23, D-8000 München 2, Germany
}

\section{$\underline{\text { ABSTRACT }}$}

For the determination of the concentration of gases by means of pressure measurement, a precise equation of state is given by which analysis can be carried out within an accuracy of $10 \mathrm{ppm}$. The parameters of the equation of state are explicitely reported for carbon dioxide, argon, and helium.

\section{INTRODUCTION}

The determination of the density or concentration of pure gases is usually carried out by a precise and 
simple pressure measurement as a standard procedure. Densities and concentrations are thereof obtained by use of an equation of state. The precision of this determination method is generally limited by the validity of the equation. Gases with low densities are precisely described by the ideal gas law. However, an equation of general validity doesn't exist for gases with high densities ${ }^{1-4}$.

\section{RESULTS AND DISCUSSION}

In other context we found, that pure gases form three different structures with increasing density: at low density a structure with nearly free motion of the particles, at higher density a structure which is characterized by the contact of particles, and at high density a densely packed state. The transition between these structures is rather abrupt. Therefore, gases are described best by three different equations corresponding to the three different structures.

For the concentration region $I$, at low densities, the pressure as a function of concentration is described best by equation (1), in which $c$ is the molar

$$
\begin{aligned}
& p=F_{p} \cdot \ln \left(c+c^{*}\right)+p^{\prime} \\
& \ln \left(p+p^{*}\right)=F_{c} \cdot c+c^{0}
\end{aligned}
$$


concentration of the gas (the density can be calculated thereof in the usual manner). $p$ is the pressure and $F_{p}$ and $c^{*}$ are the parameters of the equation. The additional parameter $\mathrm{p}^{\prime}$ is zero in region $I$. The precise validity of eq. (1) is demonstrated by fig. 1 in which a linear correlation between $p$ and In $\left(\mathrm{c} / \mathrm{c}^{*}+1\right)$ is shown for $\mathrm{CO}_{2}$ at $75.260^{\circ} \mathrm{C}$ on the basis of the precise measurements of A.Michels and C.Michels ${ }^{5}$. A straight line is obtained with a correlation factor of 0.999998 (10 points). The $\mathrm{F}_{\mathrm{p}}$ value is 133.4 at and the $c^{*}$ value $4.59 \mathrm{~mol} \cdot \mathrm{l}^{-1}$. The latter is calculated by an iterative procedure.

Eq. (1) holds up to a critical concentration $c_{k}(I-I I)$ of $3.1 \mathrm{~mol} \cdot 1^{-1}$. With higher concentrations, there is an abrupt change of medium structure. In this case (region II) eq. (1) holds too, but with different parameters $F_{p}$ and $c^{*}$, and the $p^{\prime}$ value is not zero. At the even higher concentration $c_{k}$ (II-III) of $10 \mathrm{~mol} \cdot \mathrm{I}^{-1}$ a second change in medium structure is observed. This high density region III is described by eq. (2). $F_{C^{\prime}} p^{*}$, and $c^{0}$ are the parameters of the equation. The formation of a liquid phase is due to a limited miscibility between the structures of region II and III. Therefore, the equations hold until the second phase is formed. On the basis of these results the complete diagram of the liquid and gaseous state of 


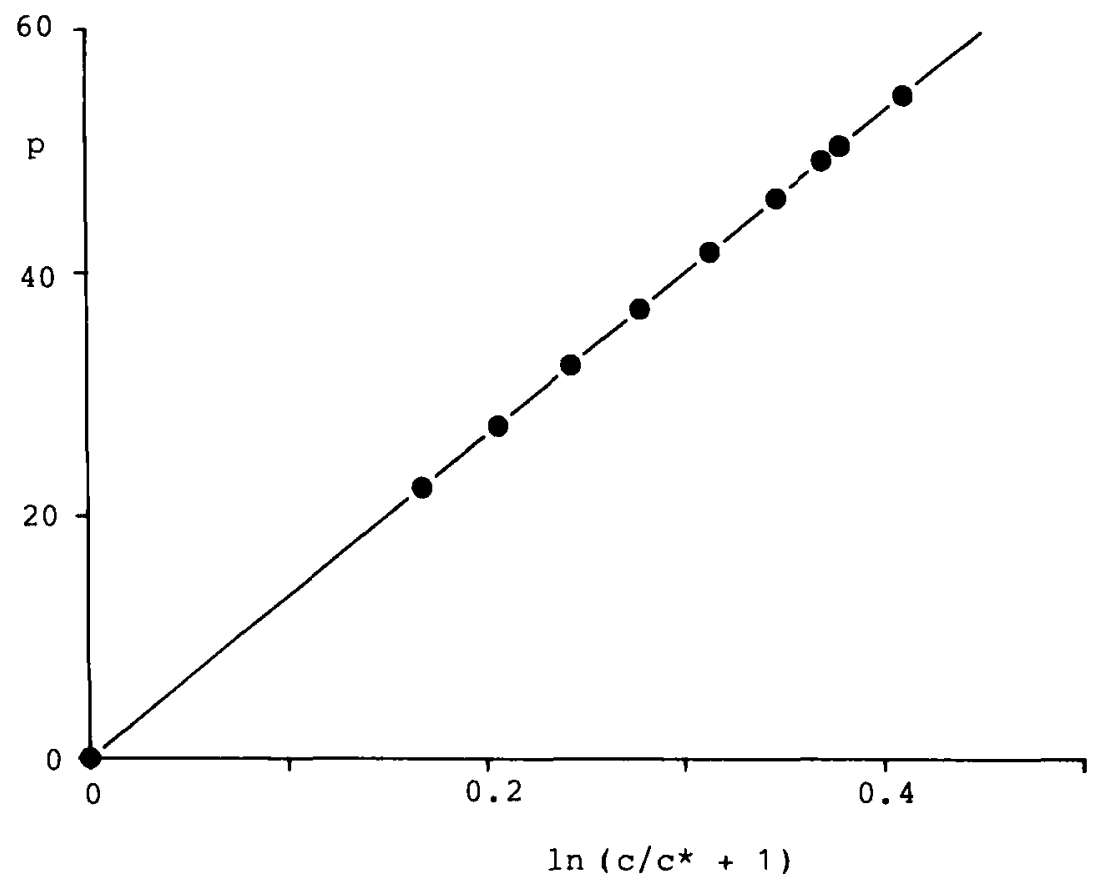

FIG. 1: Linear correlation between $\mathrm{p}$ and $\ln \left(\mathrm{c} / \mathrm{c}^{\star}+{ }^{1}\right)$ according to eq. (1) $\left(\mathrm{CO}_{2}\right.$ at $\left.75.260^{\circ} \mathrm{C}, \mathrm{c}<3.1 \mathrm{~mol} \cdot \mathrm{l}^{-1}\right)$.

$\mathrm{CO}_{2}$ can be drawn, as shown in fig. 2. The parameters of the eq. (1) and (2) are given in Table 1. The gases argon and helium are described in the same manner as carbondioxide - see Tables 2 and 3 .

\section{TEMPERATURE DEPENDENCE OF PARAMETERS}

The $c_{k}$-values are essentially independent of temperature. For the other parameters a linear interpolation between the experimental data of Tables 1 to 3 


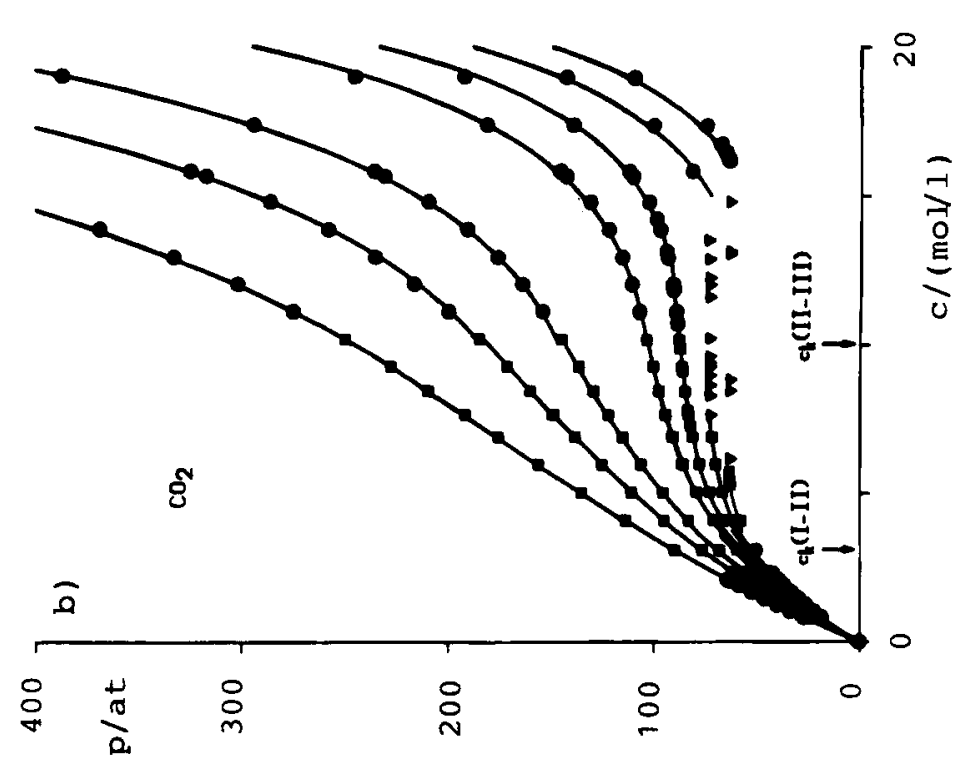

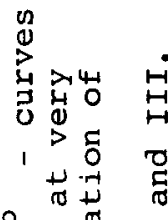

岑艺号兌

E导元

융

4 난

被

(1) ब

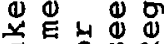

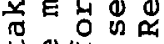

पै 4 U

1 豆

4 ㄷㅇ

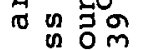

蝶

芹岳苗

包苗

包 0 \%

4 0 至

कै न के व

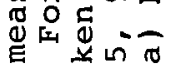

. $\frac{1}{\sigma}$

$\phi \doteq$

듬ㅇ.

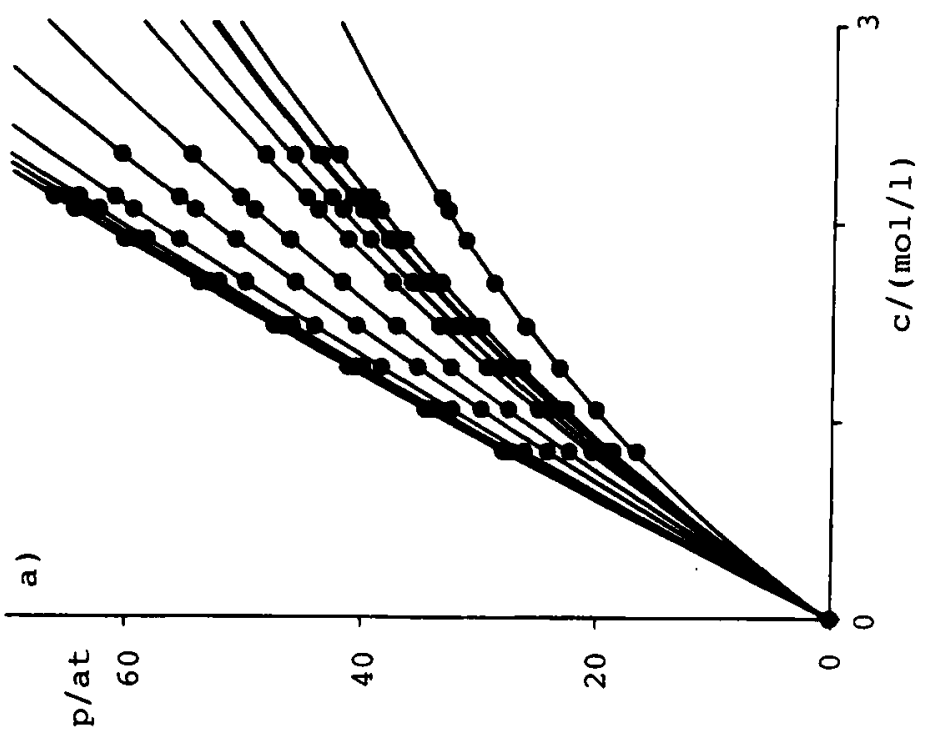

约是

题

4000

$1-5^{0}$ o

$1=0$ $0^{N} \cdot{ }^{N}$ ○通的 出 $N$ (1) 0 它苴 $\stackrel{0}{\uplus}$ 屾先 त且山 1 $+\pi-1)$ on $E$ 出 140

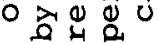
䎡它 -

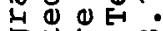
G山 $m$

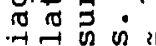

台劣行

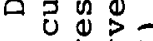
펌대 U 01 ( $)$ ही 号 


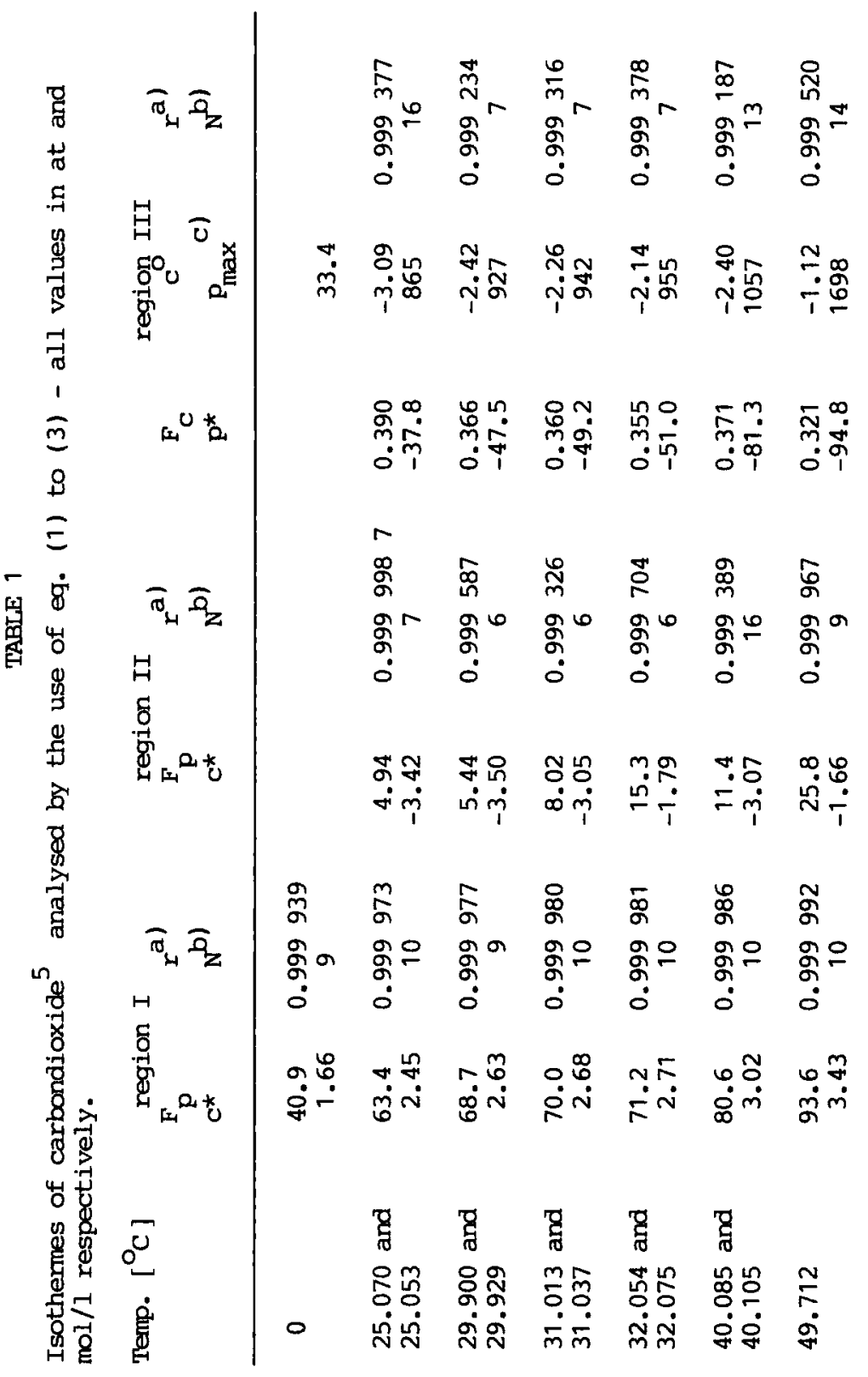




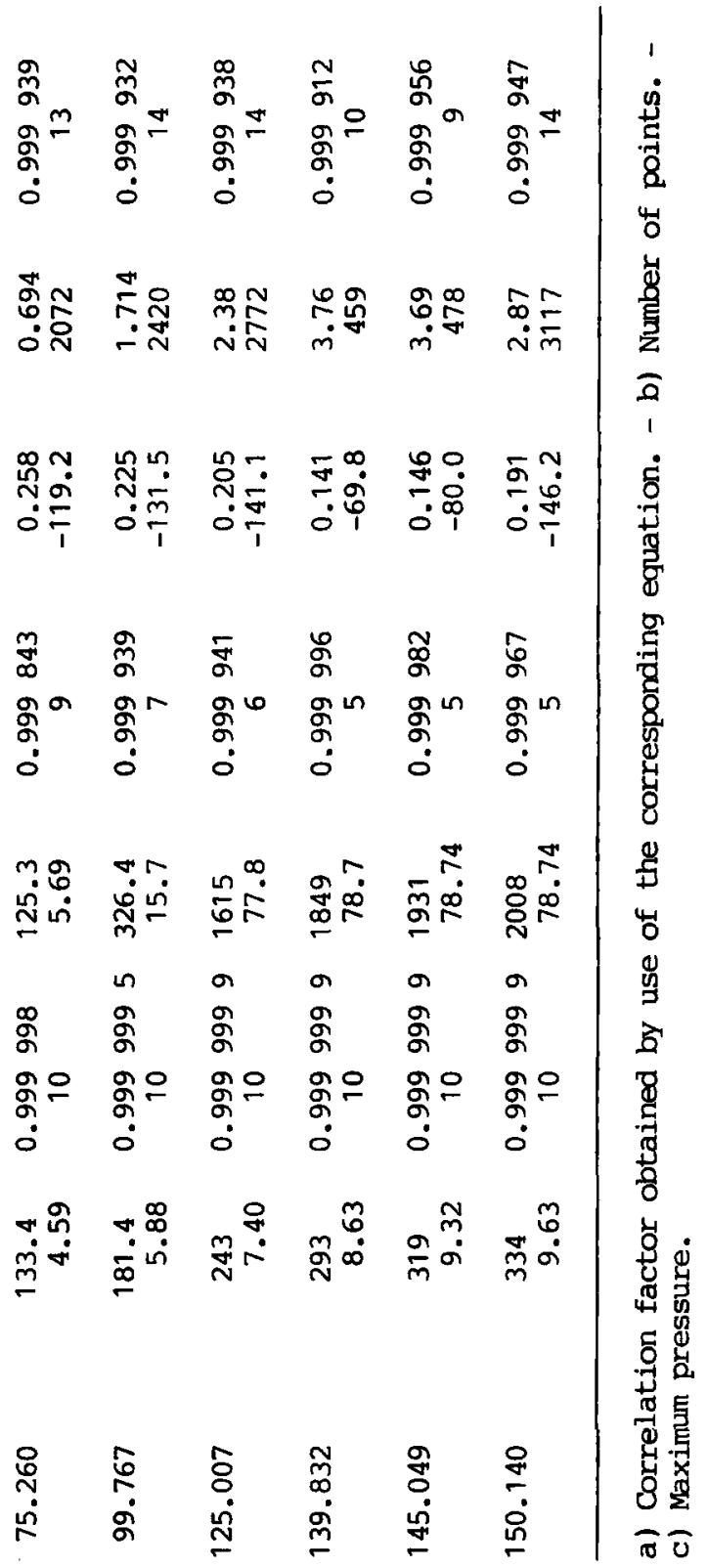




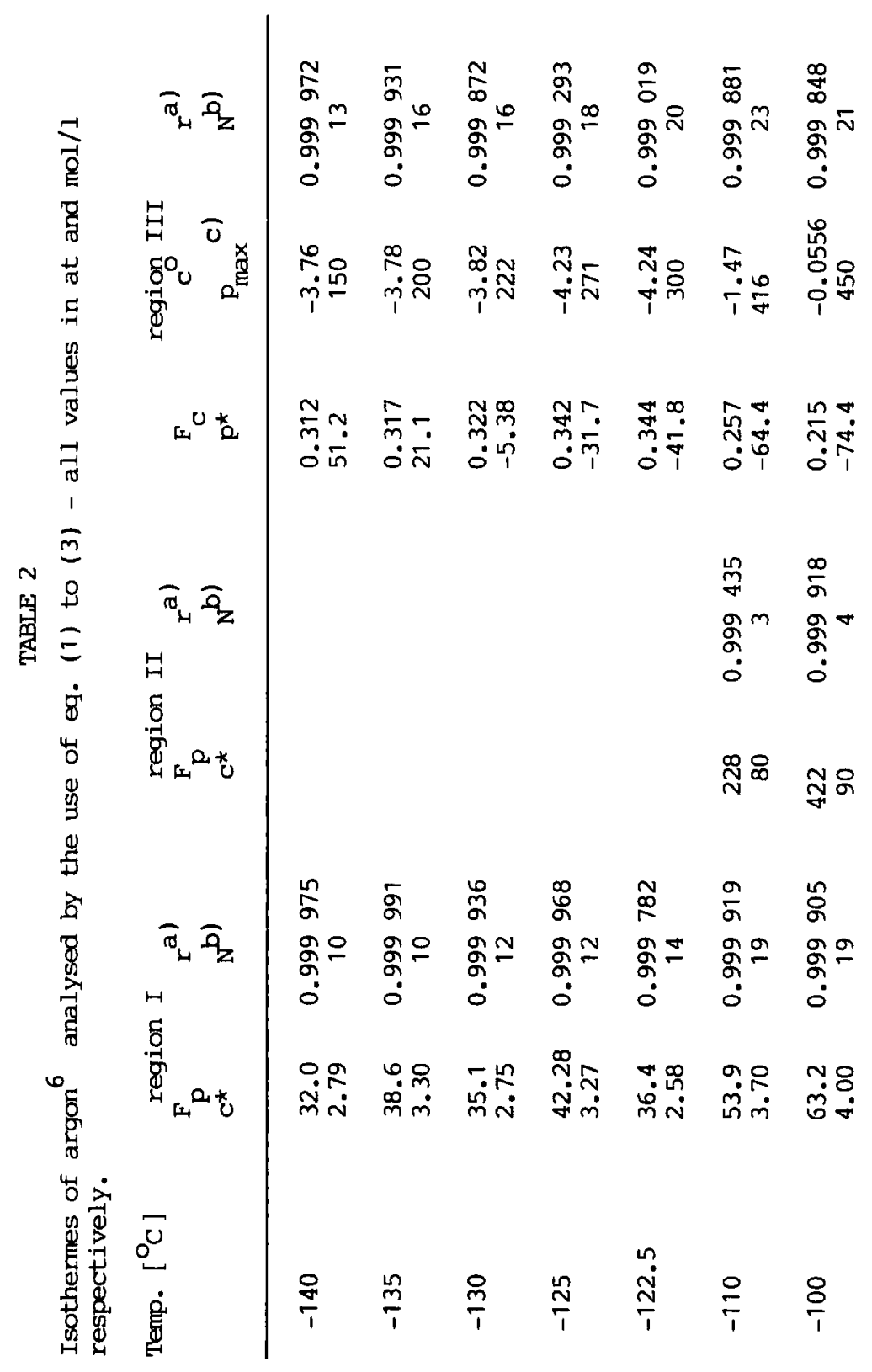




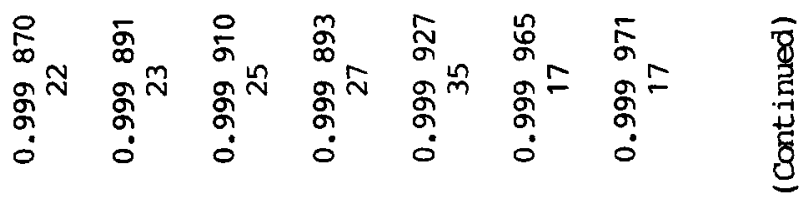

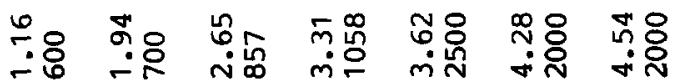

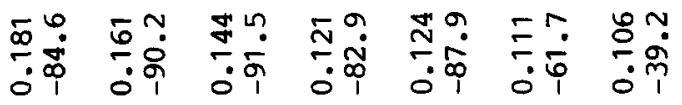

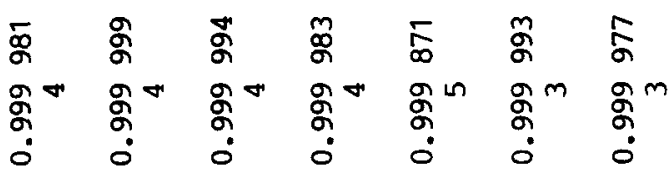

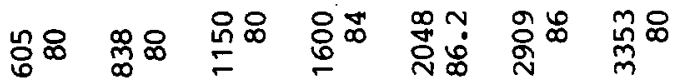

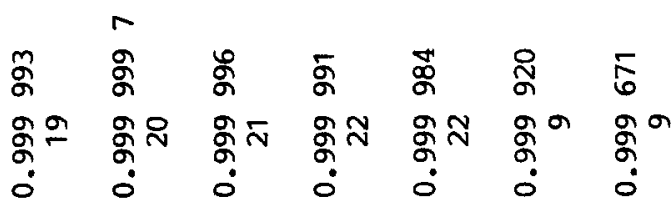

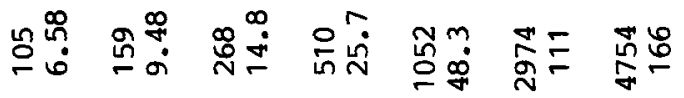

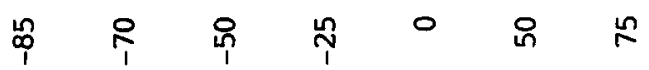




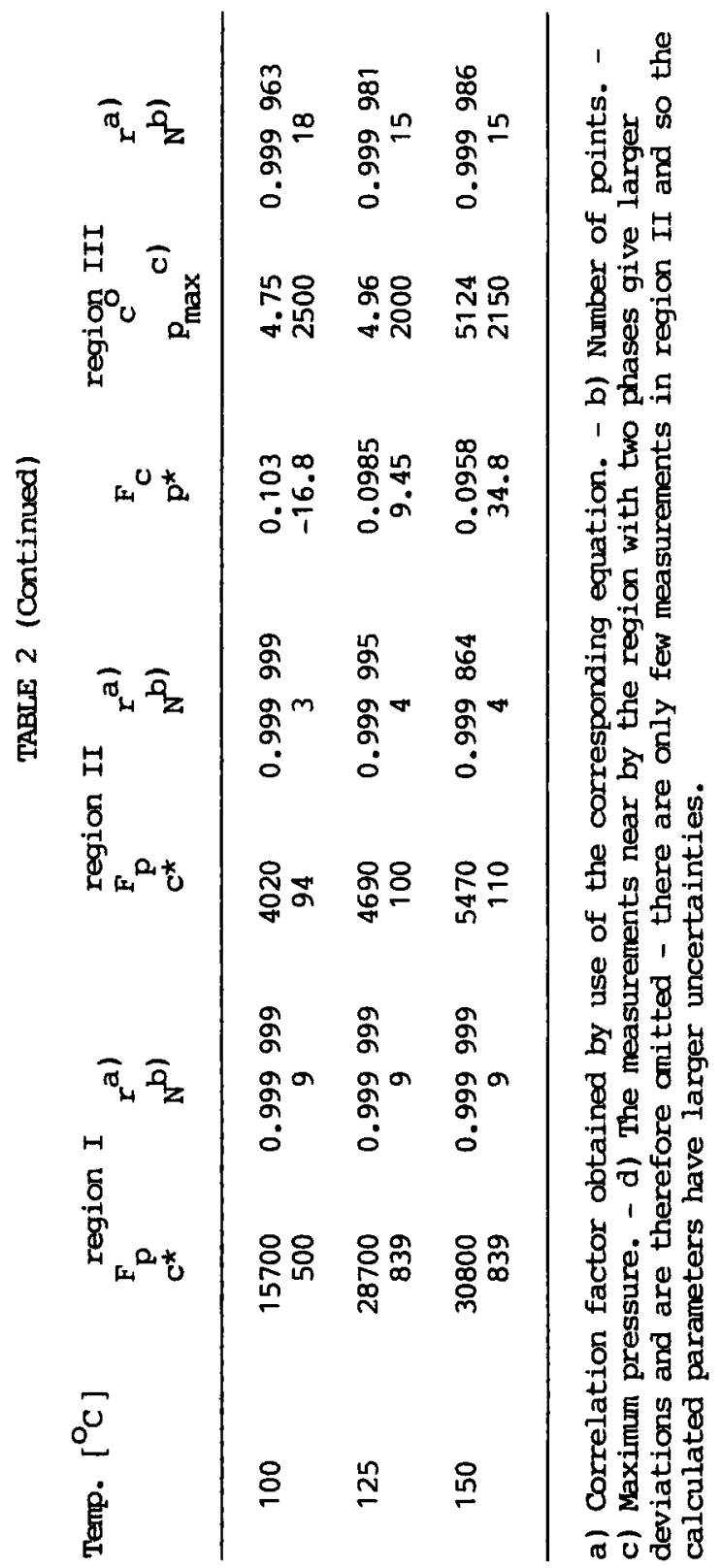


TABLE 3

Isothermes of helium ${ }^{9}$ analysed by use of eq. (1) - all values in at and mol/l, respectively.

\begin{tabular}{|c|c|c|c|c|c|}
\hline Temp. [K] & $F_{p}$ & $c^{\star}$ & $\mathrm{r}^{\mathrm{a})}$ & $N^{(b)}$ & $\mathrm{p}_{\max } \mathrm{c}$ \\
\hline $\begin{array}{l}2.610 \\
3.105 \\
3.721 \\
4.245\end{array}$ & $\begin{array}{l}0.623 \\
0.862 \\
1.113 \\
1.328\end{array}$ & $\begin{array}{l}2.87 \\
3.34 \\
3.51 \\
3.56\end{array}$ & $\begin{array}{l}0.999 \\
0.991 \\
0.999999 \\
0.999984 \\
0.999916\end{array}$ & $\begin{array}{r}18 \\
6 \\
8 \\
19\end{array}$ & $\begin{array}{l}0.116 \\
0.245 \\
0.548 \\
0.954\end{array}$ \\
\hline
\end{tabular}

a) Correlation factor obtained by the use of eq. (1). - b) Number of points. - c) Maximum pressure.

is sufficiently accurate for most applications. If even higher precision is required, the proportionality of the parameters with a power of the absolute temperature $\mathrm{T}$ can be used. This is explicitely done for $\mathrm{CO}_{2}$ in region $\mathrm{I}$, where the parameters can be determined with high precision. The temperature dependence is given by eq. (3) and (4). The constants F

$$
\begin{aligned}
& F_{p}=a_{F} \cdot T^{\alpha F} \\
& c^{\star}=a_{c} \cdot T^{\alpha C}
\end{aligned}
$$

and $\alpha c$ are obtained by plots of $\ln F_{p}$ vers. In $T$ and 
In $c^{*}$ vers. In $T$. Plots of $F_{p}$ vers. $T^{\alpha F}$ and $c^{\star}$ vers. $\mathrm{T}^{\alpha C}$ are given by fig. 3. Straight lines are obtained with correlation factors $r=0.9997$ and $r=0.9995$ $(13$ points). The intercepts are very small and can be omitted in most cases. The temperature dependence of the parameters in region II and III can be described in the same manner as in region $I$ with the same type of equations as (3) or (4).

DETERMINATION OF CRITICAL CONCENTRATIONS $c_{k}$

The determination of $c_{k}(I-I I)$ and $c_{k}(I I-I I I)$ is important for the practical use of eq. (1) and (2). The clue for the determination of $c_{k}$ is the fact that the correlation according to either eq. (1) or (2) rapidly turns bad when a $c_{k}$ value is exceeded. This is shown in fig. 4 where the correlation factor is plotted vers. the highest concentration which is taken into account. The point of inflection in fig. 4 gives a $c_{k}$ (I-II) value of $3.1 \mathrm{~mol} \cdot \mathrm{I}^{-1}$. The $c_{k}$ (II-III) can be obtained in the same manner starting with the highest concentration and going stepwise to lower concentrations. The so obtained $c_{k}$ values can be verified by the analogous use of region II.

The point of inflection is more pronounced at lower temperatures, but can easily be localized even at high temperatures. Measurements with lower accuracy give the same type of plot as fig. 4 . 

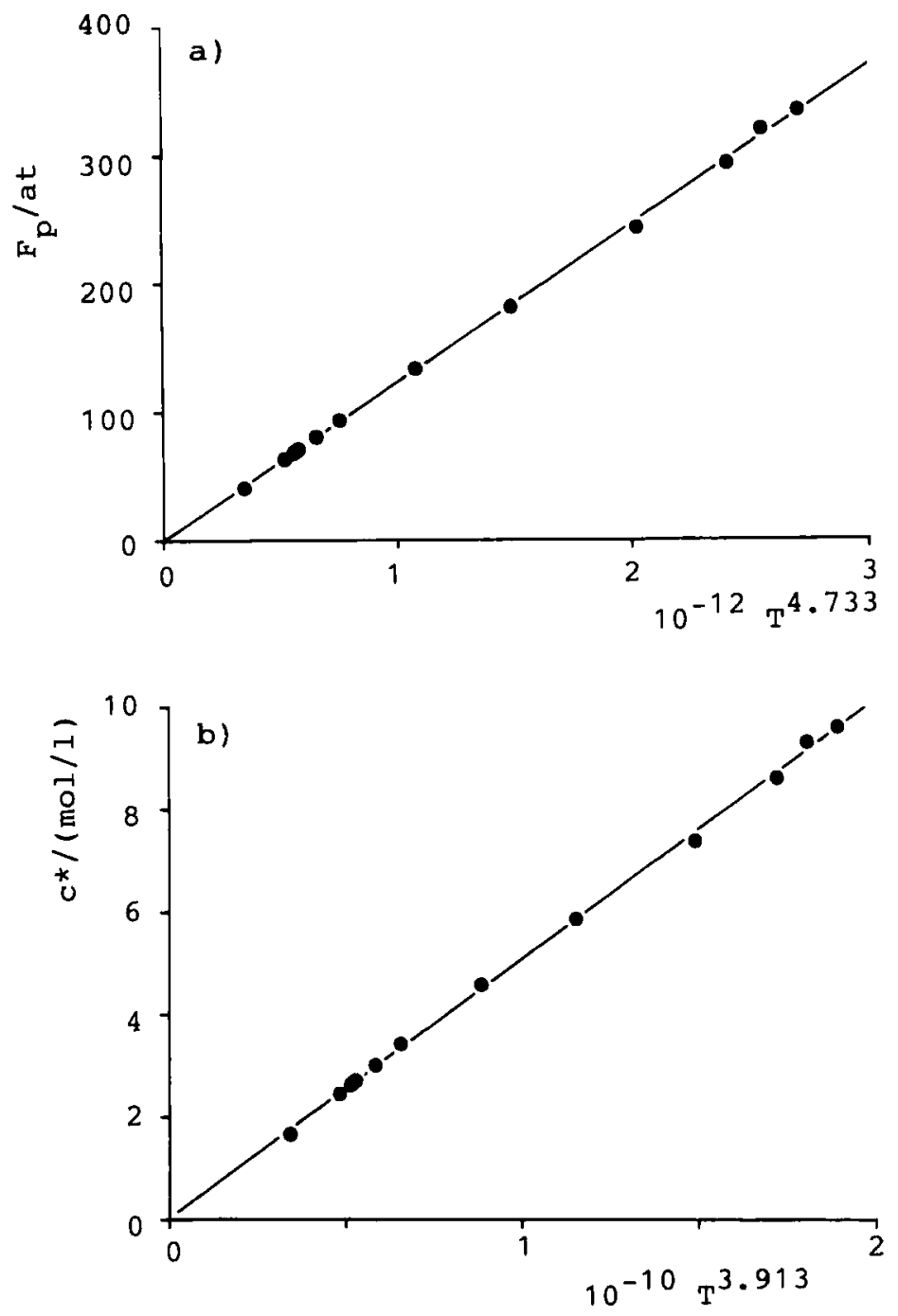

FIG. 3: Temperature dependence of the parameters $F_{p}$ and $c *$ of carbon dioxide, region $I$. - a) Plot of $F_{p}$ vers. $\mathrm{T}^{4.73}\left(\mathrm{a}_{\mathrm{F}}=1.230 \cdot 10^{-10}\right.$, intercept: $\left.0.049 \mathrm{at}\right)$. $^{\mathrm{p}}$ b) Plot of $c^{\star}$ vers. $T^{3.913}\left(a_{c}=5.049 \cdot 10^{-10}\right.$, intercept: $0.036 \mathrm{~mol} \cdot \mathrm{l}^{-1}$ ). 


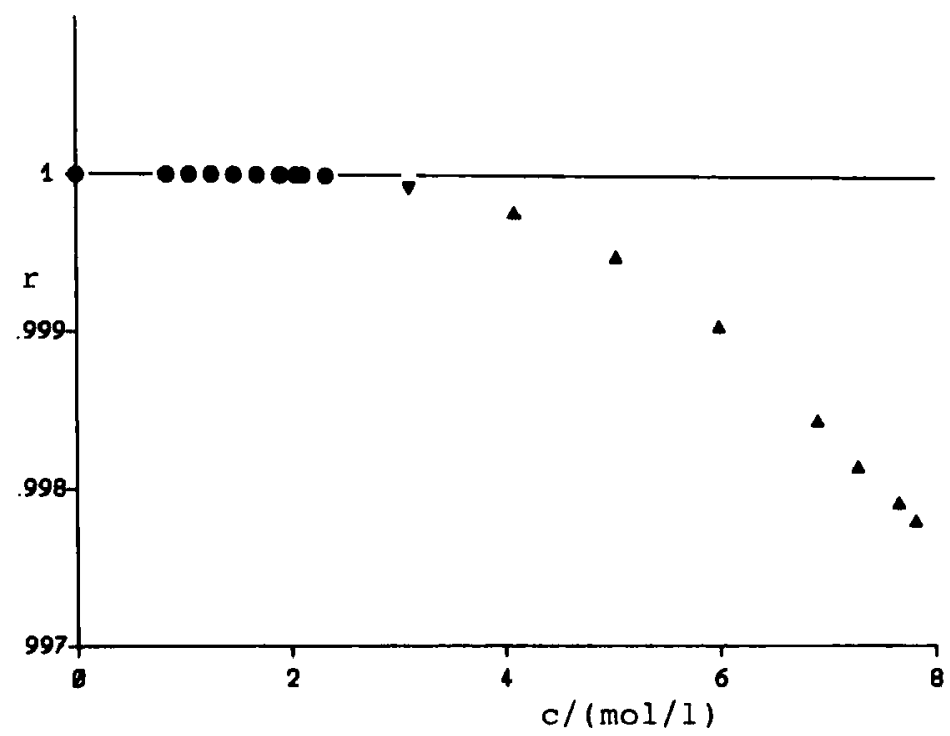

FIG. 4: Correlation factor as a function of the highest concentration in the plot of $p$ vers. In $\left(c / c^{*}+1\right)$ for region I of carbondioxide at 40.105 and $40.085^{\circ} \mathrm{C}^{5}$.

USE OF THE EQUATIONS FOR ANALYSIS

For the calculation of the concentrations eq. (1)

and (2) should be transformed to eq. (5) to (7) which

$$
\begin{array}{ll}
c=c^{\star} \cdot \exp \left(p / F_{p}\right)-c^{*} & \text { (5) Region I } \\
c=c^{*} \cdot \exp \left(\frac{p-p^{\prime}}{F_{p}}\right)-c^{*} & \text { (6) Region II } \\
c=\left(\ln \left(p+p^{\star}\right)-c^{0}\right) / F_{c} & \text { (7) Region III }
\end{array}
$$


directly give the concentration $c$. When a concentration is to be calculated one has to check to which region the pressure measurement corresponds. Then the parameters of the equations are taken from table 1 to 3 , for other temperatures interpolated as described, or calculated by use of eq. (3) and (4). With a linear interpolation a sufficient accuracy in most cases is obtained. For other gases the parameters of eq. (1) and (2) can be calculated in the described manner.

\section{EXPERIMENTAL EXAMPLE}

The concentration of carbondioxide is to be determined to a given pressure ${ }^{5}$ of 46.324 at $75.260^{\circ} \mathrm{C}$. The pressure corresponds to region $I$. The parameters $F_{p}=133.4$ at and $c^{\star}=4.59 \mathrm{~mol} \cdot 1^{-1}$ for this temperature are taken from Table 1. By use of eg. (5) a concentration $c$ of $1.9569 \mathrm{l}, \mathrm{ol} \cdot \mathrm{I}^{-1}$ is calculated, whereas the experimental concentration is $1.9567 \mathrm{~mol} \cdot \mathrm{l}^{-1}$. The error is $10 \mathrm{ppm}$. The molar concentration can be transferred into density or molar volume in known manner.

\section{ACKNOWLEDGEMENT}

This work was supported by Deutsche Forschungsgemeinschaft. 


\section{REFERENCES}

1. G.C.Maitland, M.Rigby, E.B.Smith, and W.A.Wakeham, Intermolecular Forces, 1.edition, Clarendon Press, Oxford 1981.

2. J.O.Hirschfelder, C.F.Curtis und R.B.Bird, Molecular Theory of Gases and Liquids, 2.edition, John Wiley \& Sons, New York 1964.

3. G.Kortüm, Einführung in die Chemische Thermodynamik, 6.edition, Verlag Chemie GmbH, Weinheim 1972.

4. J.otto in Landolt - Börnstein Zahlenwerte und Funktionen aus Physik Chemie Astronomie Geophysik Technik; 1.edition, vol. II/1, p. 298, springer verlag, Heidelberg 1971 .

5a. A.Michels and C.Michels, Proc.Roy.Soc.London, Ser.A, 153 (1935) 201.

5b. A.Michels, C.Michels, and H.Wouters, Proc.Roy.Soc. London, Ser.A, 153 (1935) 214.

5c. A.Michels, B.Blaisse, and C.Michels, Proc.Roy.Soc. London, Ser.A, 160 (1937) 358.

6a. A.Michels, J.M.Levelt, and G.J.Wolkers, Physica 24 (1958)769.

6b. A.Michels, J.M.Levelt, and W.de Graaff, Physica $24,659(1958)$.

6c. A.Michels, H.Wijker, and H.K.Wijker, Physica 15 (1949) 627 .

6d. A.Michels, R.J.Lunbeck, and G.J.Wolkers, Appl. Scient.Res.A 2 (1951) 345 .

7a. W.H.Keesom and H.K.Kraak, Physica 2 (1935) 37.

7b. W.H.Keesom and W.K.Walstra, Physica $\underline{7}$ (1940) 985 . 